\title{
Volatile affairs in microbial interactions
}

\author{
Ruth Schmidt ${ }^{1,5}$, Viviane Cordovez ${ }^{1,2,5}$, Wietse de Boer ${ }^{1,3}$, Jos Raaijmakers ${ }^{1,4}$ \\ and Paolina Garbeva ${ }^{1}$ \\ ${ }^{1}$ Department of Microbial Ecology, Netherlands Institute of Ecology (NIOO-KNAW), Wageningen, \\ The Netherlands; ${ }^{2}$ Laboratory of Phytopathology, Wageningen University, Wageningen, The Netherlands; \\ ${ }^{3}$ Department of Soil Quality, Wageningen University, Wageningen, The Netherlands and ${ }^{4}$ Institute of \\ Biology (IBL), Leiden University, Leiden, The Netherlands
}

\begin{abstract}
Microorganisms are important factors in shaping our environment. One key characteristic that has been neglected for a long time is the ability of microorganisms to release chemically diverse volatile compounds. At present, it is clear that the blend of volatiles released by microorganisms can be very complex and often includes many unknown compounds for which the chemical structures remain to be elucidated. The biggest challenge now is to unravel the biological and ecological functions of these microbial volatiles. There is increasing evidence that microbial volatiles can act as infochemicals in interactions among microbes and between microbes and their eukaryotic hosts. Here, we review and discuss recent advances in understanding the natural roles of volatiles in microbe-microbe interactions. Specific emphasis will be given to the antimicrobial activities of microbial volatiles and their effects on bacterial quorum sensing, motility, gene expression and antibiotic resistance.
\end{abstract}

The ISME Journal (2015) 9, 2329-2335; doi:10.1038/ismej.2015.42; published online 29 May 2015

\section{Introduction}

Microorganisms from diverse ecosystems produce a wide range of volatile organic compounds. Compared with other secondary metabolites (for example, enzymes, antibiotics and toxins), volatiles are typically small compounds (up to C20) with low molecular mass (100-500 Daltons), high vapour pressure, low boiling point and a lipophilic moiety. These properties facilitate evaporation and diffusion through both water- and gas-filled pores in soil and rhizosphere environments. Hence, microbial volatiles have important roles in marine and terrestrial environments (Schulz et al., 2010; Romoli et al., 2014). To date, the chemical structure of $\sim 1000$ volatiles have been described originating from a wide range of bacterial and fungal genera and species (Effmert et al., 2012; Lemfack et al., 2014). Bacterial volatiles are typically dominated by alkenes, alcohols, ketones, terpenes, benzenoids, pyrazines, acids and esters, whereas fungal volatiles are dominated by alcohols, benzenoids, aldehydes, alkenes, acids, esters and ketones (Piechulla and Degenhardt, 2014). Most microbial volatiles are considered as sideproducts of primary and secondary metabolism. They are formed mainly by oxidation of glucose

Correspondence: P Garbeva, Department of Microbial Ecology, Netherlands Institute of Ecology (NIOO-KNAW), Droevendaalsesteeg 10, PO Box 50, 6708 PB Wageningen, The Netherlands.

E-mail: p.garbeva@nioo.knaw.nl

${ }^{5}$ These authors contributed equally to this work.

Received 4 December 2014; revised 15 February 2015; accepted 23 February 2015; published online 29 May 2015 from various intermediates (Korpi et al., 2009). The underlying biosynthetic pathways are aerobic, heterotrophic carbon metabolism, fermentation, amino-acid catabolism, terpenoid biosynthesis, fatty acid degradation and sulphur reduction (Peñuelas et al., 2014). The main metabolic pathways for microbial volatiles are summarised in Figure 1.

Although there are common volatiles produced by different, often unrelated, microorganisms, other volatiles are unique for certain strains (Schulz and Dickschat, 2007; Garbeva et al., 2014a,b). The amount and composition of volatiles produced by microorganisms can vary according to culturing conditions (Claeson, 2007; Blom et al., 2011; Garbeva et al., 2014a,b). Other important factors influencing the production of volatiles are the physiological state of the producing microorganism, oxygen availability, moisture, temperature and $\mathrm{pH}$ (Insam and Seewald, 2010; Romoli et al., 2014).

The importance of microbial volatiles for the ecology of microorganisms has been overlooked for a long time, probably due the lack of appropriate detection techniques. However, in the last 10 years the number of studies on microbial volatiles has increased substantially in different research areas such as food, medical, agricultural and environmental sciences. In this review, we focus on the ecological role of volatiles in microbe-microbe interactions. For more information on techniques used for volatile analyses and their role in microbe interactions with their eukaryotic hosts, we refer to several recent reviews (Effmert et al., 2012; Farag et al., 2013; Junker and Tholl, 2013; Peñuelas et al., 2014). 


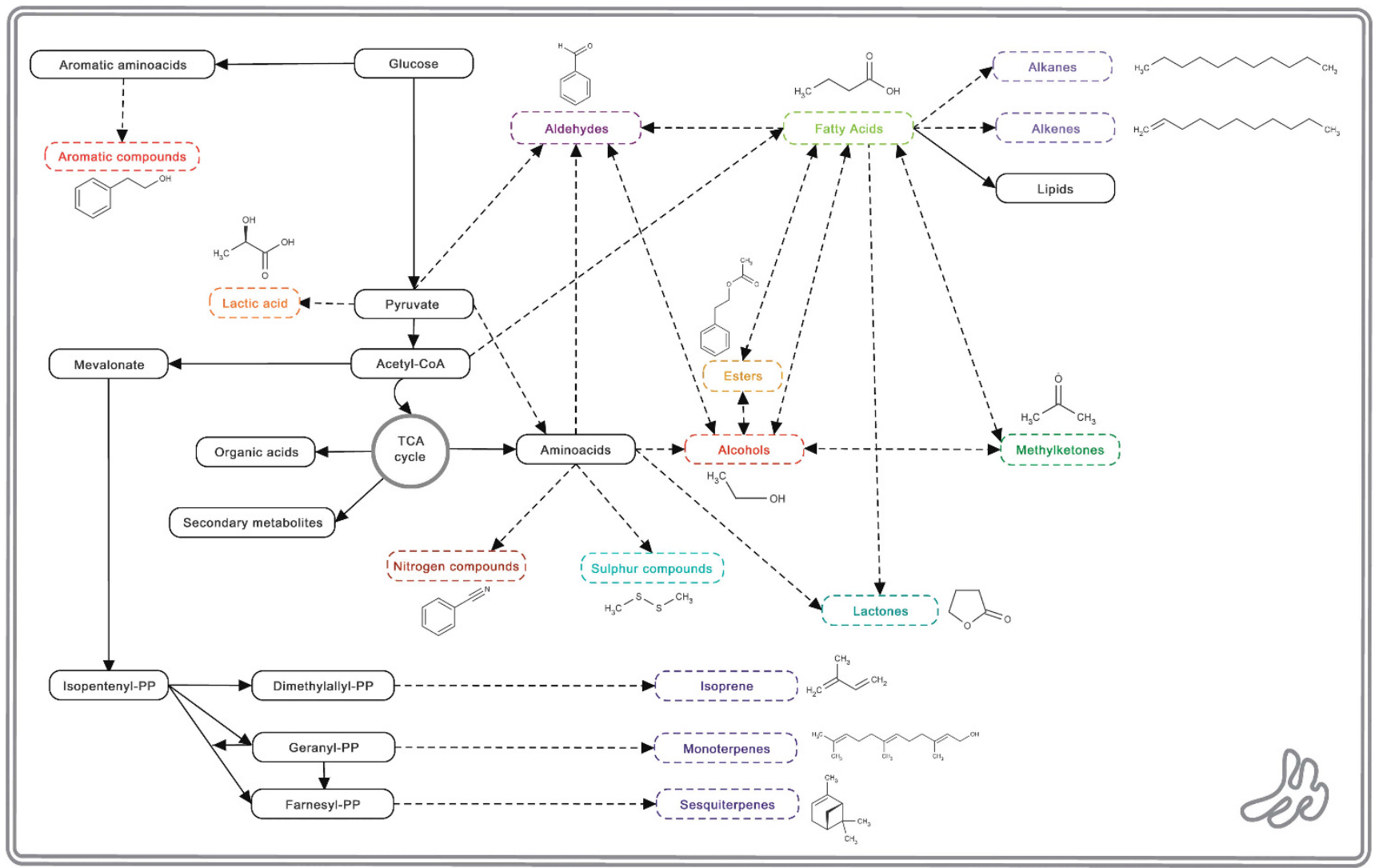

Figure 1 Main metabolic pathways for the production of microbial volatiles. Volatiles are depicted in coloured dashed rectangles indicating different chemical classes. Representative examples are given per class: alcohols (for example, ethanol), aldehydes (for example, benzaldehyde), alkanes (for example, undecane), alkenes (1-undecene), aromatic compounds (for example, 2-phenylethanol), esters (for example, 2-phenylethyl ester), fatty acids (for example, butyric acid), isoprene, lactic acid, lactones (for example, gammabutyrolactone), methylketones (for example, acetone), monoterpenes (for example, farnesol), nitrogen compounds (for example, benzonitrile), sesquiterpenes (for example, pinene) and sulphur compounds (for example, dimethyl disulphide).

\section{Ecological roles of microbial volatiles in antagonistic interactions}

Microbial volatiles can have a significant role in antagonistic interactions between microorganisms occupying the same ecological niche. Here, we will focus on the antimicrobial activity of volatiles with specific emphasis on their antifungal and antibacterial activities.

\section{Volatile-mediated antifungal activity}

It is well known that germination of fungal spores as well as hyphal growth can be inhibited by bacterial volatiles (Herrington et al., 1985, 1987). Furthermore, exposure to bacterial volatiles has been reported to change fungal morphology, enzyme activity and gene expression (Wheatley, 2002; Vespermann et al., 2007; Minerdi et al., 2008, 2009; Kai et al., 2009; Garbeva et al., 2011, 2014b). For example, activity of laccases and tyrosinases can be strongly affected by bacterial volatiles (Wheatley, 2002).

Fungal volatiles can also have inhibitory effects on other fungi. For example, the endophytic fungi
Muscodor albus and Oxysporus latemarginatus strongly inhibited growth of several plant pathogenic fungi, including Botrytis cinerea and Rhizoctonia solani (Strobel et al., 2001). Moreover, M. albus volatiles were shown to kill the fungal human pathogens Aspergillus fumigatus and Candida albicans (Strobel et al., 2001). Fungi often live in symbiosis with bacteria. For Fusarium oxysporum, hyphae-associated bacteria were shown to produce the volatile sesquiterpene caryophyllene, which repressed the expression of two virulence genes. When cured from the bacterial symbionts, caryophyllene was not detected and $F$. oxysporum became pathogenic (Minerdi et al., 2008).

Sensitivity to volatiles can strongly differ between fungal species and the extent of inhibition depends on the individual bacteria-fungus or fungus-fungus interaction (Kai et al., 2007, 2009; Vespermann et al., 2007; Garbeva et al., 2014b). Several independent studies have reported that $F$. solani is not much affected by bacterial volatiles, whereas Pythium species (oomycetes) are highly sensitive to bacterial volatiles (Kai et al., 2009; Effmert et al., 2012; Garbeva et al., 2014a,b). F. oxysporum was also reported to be rather resistant to volatiles produced 
by the fungus $O$. latemarginatus, whereas Magnaporthe grisea was sensitive. High sensitivity to bacterial volatiles was recently reported for the late blight oomycete pathogen Phytophthora infestans. Two volatiles, hydrogen cyanide and 1-undecen, were indicated as the main compounds responsible for the growth inhibition (Hunziker et al., 2015). The apparent high sensitivity of oomycetes to volatiles may be related to their cell wall composition and structure, which is different from that of fungi. To date, however, very little is known about fungal resistance to volatiles with the exception of resistance to azole-derived compounds (Lupetti et al., 2002). Azole resistance commonly involves modifications of the cyp51A gene, the target of antifungal azoles (Lupetti et al., 2002, Seyedmousavi et al., 2014). The resistance selection is believed to occur via exposure to azole compounds in the environment (Snelders et al., 2009), released by humans via application of crop protections agents or by bacterial genera commonly found in soil, such as Bacillus, Serratia, Pseudomonas and Burkholderia (Lemfack et al., 2014).

\section{Volatile-mediated antibacterial activity}

Relatively few studies have reported on volatiles with antibacterial activity. Screenings of commonly produced volatiles with antimicrobial activity often did not reveal antibacterial activity (Schulz et al., 2010). Moreover, volatiles with strong antifungal activity (such as dimethyl disulphide, dimethyl trisulphide, $S$-methyl thioacetate and benzonitrile) did not exhibit antibacterial effects and even stimulated the growth of some bacteria (Garbeva et al., 2014a). However, some specific volatiles produced by only a few microorganisms have been indicated as potential antibacterial agents. These include volatile lactones such as $\gamma$-butyrolactones, which exhibit antibacterial activity against a broad range of Gram-positive and Gramnegative bacteria (Schulz et al., 2010).

An odoriferous actinomycete isolate from corn seeds, identified as Streptomyces albidoflavus, was shown to produce a sesquiterpene, named albaflavenone with antibacterial properties (Gurtler et al., 1994). More recently, albaflavenone was isolated from other Streptomyces species and fungi (Takamatsu et al., 2011; Moody et al., 2012). Another sesquiterpene compound with antibacterial activity, dihydro- $\beta$-agarofuran, is produced by Streptomyces sp. (Brana et al., 2014). Recently, Dandurishvili et al. (2011) reported that volatiles emitted by Pseudomonas fluorescens and Serratia plymuthica have bacteriostatic effects against the bacterial plant pathogens Agrobacterium tumefaciens and A. vitis and inhibited the growth of these pathogens in planta. The major volatile emitted by $S$. plymuthica under the tested conditions was dimethyl disulphide, whereas $P$. fluorescens emitted a mix of 1-undecene, methanthiol, methanthiol acetate and dimethyl disulphide (Dandurishvili et al., 2011).
Volatile-producing endophytes have recently attracted great attention due to their strong antimicrobial activity. For example, $M$. albus (an endophytic fungus of tropical tree species) emitted a number of volatiles, such as tetrohydofuran, aciphyllene and an azulene derivate (Atmosukarto et al., 2005). Volatiles emitted by M. albus as well as the artificial mixture of volatiles effectively inhibited or killed a range of plant and human-pathogenic bacteria. Another recently described endophytic fungus $M$. crispans, isolated from wild pineapple, produced a mixture of volatile compounds with strong activity against a major bacterial pathogen of citrus, Xanthomonas axonopodis pv. citri, and the human pathogens Yersinia pestis, Mycobacterium tuberculosis and Staphylococcus aureus (Mittchell et al., 2010). Five classes of volatiles (acids, alcohols, esters, ketones and lipids) were identified in Muscodor species and although each class had some antimicrobial effect, their collective action was required to kill a broad range of bacterial pathogens.

In the past years, a group of pyrazine volatile compounds have been attracting wide interest due to their promising antitumour, antimicrobial and insecticidal activities (Rajini et al., 2011). The production of pyrazines is widely distributed in plants, and only few bacteria have been reported so far to synthesize these volatile compounds (Rajini et al., 2011; Brana et al., 2014). S. albus, Corynebacterium glutamicum and Bacillus spp. produce tetramethylpyrazine (also known as ligustrazine), a compound that is used in traditional Chinese medicine against cystic fibrosis.

Although the mode of action of antibacterial volatiles has not been studied in detail, it is likely that hydrophobicity of some volatiles enables them to partition in the lipid layer of the cell membrane, rendering the membrane more permeable. Indeed, a study on the mechanisms of inhibitory action of three monoterpenes against S. aureus and Escherichia coli revealed a perturbation of the lipid fraction of microorganisms' plasma membrane, resulting in alteration of membrane permeability and a leakage of intracellular compounds (Trombetta et al., 2005).

Finally, volatile compounds may have a synergistic effect when combined with antibiotics. For example, hydrophilic antibiotics such as vancomycin and $\beta$-lactam antibiotics, which have a marginal activity on the Gram-negative bacteria E. coli and Listeria monocytogenes, exhibit an enhanced antibacterial activity when pre-treated with the volatile eugenol (Hemaiswarya and Doble, 2010). Synergistic effects of terpenes and penicillin on multiresistant strains $S$. aureus and E. coli have also been reported (Gallucci et al., 2009).

\section{Ecological role of microbial volatiles in interspecific interactions}

Volatiles have an important role in interactions between physically separated microorganisms. 
Microarray analysis of E. coli exposed to volatiles emitted by Bacillus subtilis revealed that volatiles induce changes in gene expression and affect motility and biofilm formation of the exposed bacteria (Kim and Ryu, 2013). More recently, a study, using P. putida as a model organism, showed that indole has a role as an interspecific signalling molecule (Molina-Santiago et al., 2014). This compound influenced the expression pattern of $P$. putida genes involved in cell metabolism, cell wall biosynthesis and stress defence. In our research group, we have tested the effect of volatiles emitted by different soil bacteria grown in sand supplemented with artificial root exudates on the soil bacterium $P$. fluorescens. The $P$. fluorescens strain was grown on nutrient-limited agar while being exposed to volatiles produced by four phylogenetically different bacterial isolates (Collimonas pratensis, S. plymuthica, Paenibacillus sp. and Pedobacter sp.) as well as a mixture of all four bacteria. A genome-wide, microarray-based analysis revealed that volatiles of each bacterial strain affected gene expression of $P$. fluorescens, but with a different pattern for each strain. Only a small core set of 22 genes was differentially expressed by all volatileproducing bacteria, including the mixture. These genes were mainly involved in amino-acid transport and metabolism, energy production and conversion, signal transduction mechanisms, inorganic ion transport and metabolism, secretion and cell motility. Among these common, differentially expressed genes was the Pfl_0064 catalase, an important enzyme that protects the cell against damage by reactive oxygen species (Lushchak, 2001). Furthermore, the volatiles produced by $C$. pratensis triggered the production of antimicrobial secondary metabolites (Garbeva et al., 2014a).

Antibiotic production triggered by volatiles in microbial interactions was also observed in $P$. aeruginosa during co-culture with Enterobacter aerogenes and this enhanced production was due to the volatile 2,3-butanediol emitted by $E$. aerogenes (Venkataraman et al., 2014). Also for Chromobacterium violaceum and $P$. aeruginosa, several monoterpenes increased violacein and pyocyanin production, respectively (Ahmad et al., 2014). The fact that the production of antibiotics in these bacteria is regulated by quorum sensing (QS) suggests that volatiles may interfere with bacterial cell-cell communication. Indeed, several studies revealed that volatiles can affect QS systems in bacteria, negatively or positively (Schulz et al., 2010; Chernin et al., 2011; Ahmad et al., 2014). For example, volatiles produced by S. plymuthica can inhibit cell-cell communication mediated by acyl homoserine lactone molecules in Agrobacterium, Pectobacterium and Pseudomonas. Volatiles emitted by $S$. plymuthica decreased the amount of acyl homoserine lactone produced by these bacteria, leading to significant suppression of transcription of acyl homoserine lactone synthase genes (Chernin et al., 2011).
Volatiles may also influence fungal QS as well as fungal development and virulence. C. albicans and C. dubliniensis, well-known human opportunistic pathogenic yeasts, produce large amounts of the QS molecule (E,E)-farnesol, a sesquiterpene, that is able to modulate morphogenesis of these species. Accumulation of farnesol blocked the yeast-tomycelium morphology switch, mycelial development and biofilm formation, important traits for virulence of Candida (Hornby et al., 2001; Martins et al., 2007). Moreover, volatiles produced by Trichoderma were shown to function as signalling molecules regulating development and mediating intercolony communication: volatiles such as 1-octen-3-ol, 3-octanol and 3-octanone produced by conidiating colonies elicited conidiation in other colonies (Nemcovic et al., 2008). The underlying mechanisms of the effects volatiles on fungal development remain largely unknown.

Recently, several studies reported on the effect of volatiles on bacterial antibiotic resistance or tolerance. For example, exposure of E. coli to volatiles emitted by Burkholderia ambifaria increased its resistance to gentamycin and kanamycin by yet unknown mechanisms (Groenhagen et al., 2013). Exposure to the volatile compound trimethylamine was shown to modify the antibiotic resistance profiles of several Gram-positive and Gram-negative bacteria (Létoffé et al., 2014). In addition, indole, a volatile that has been proposed to act as signalling molecule, can also affect antibiotic resistance. For example, $P$. putida does not produce the volatile indole itself but recognizes indole produced by other bacteria (for example, E. coli) and activates the expression of the gene encoding the TtgGHI efflux pump (Lee et al., 2010; Molina-Santiago et al., 2014). Biogenic ammonia, an inorganic volatile compound, was also reported to modify antibiotic resistance in physically separated bacteria (Bernier et al., 2011). One of the underlying mechanisms proposed involves ammonia-induced synthesis of polyamines, which alters the permeability of the bacterial membrane or helps the bacteria to cope with oxygen radicals. A recent study reported on ammoniamediated growth promotion of ampicillin-sensitive bacteria by means of antibiotic inactivation (Cepl et al., 2014). However, this phenomenon appeared to result from $\mathrm{pH}$ increase in the media caused by bacterial volatiles rather than by alteration of specific traits in the target bacterium. Another inorganic volatile compound, hydrogen sulphide, was suggested as a universal defence against antibiotics in bacteria as it seemed to trigger broad-spectrum antibiotic resistance, most probably due to alleviation of oxidative stress (Shatalin et al., 2011).

As was shown for the above mentioned Candida species, also virulence and fitness of microorganisms can be affected by microbial volatiles. This was for instance observed for Pectobacterium species, bacterial pathogens responsible for soft rot disease in potato. Disruption of the biosynthesis of the volatile 
2,3-butanediol coincided with reduced virulence (Marquez-Villavicencio et al., 2011).

Volatile compounds can also have a role in the attraction of other microorganisms. During interaction between $X$. perforans and Paenibacillus vortex, volatiles produced by $X$. perforans were found to attract the proficient swarmer $P$. vortex (Hagai et al., 2014). Interestingly, the volatiles released by $X$. perforans did not only attract the swarmer but also increased its dispersal without affecting its growth rate. Using fluorescent-stained $X$. perforans, Hagai et al. (2014) revealed that this hitch-hiking strategy also occurs on tomato leaves with different swarming bacterial species, suggesting that this might be a widespread and ecologically important phenomenon.

\section{Conclusions and perspectives}

Most studies to date have focused on the role of volatiles in plant-microbe interactions and their role in plant growth and health (Bitas et al., 2013; Peñuelas et al., 2014). However, the role of volatiles in microbe-microbe communication and competition in soils remains largely unknown. It is not completely clear why microorganisms produce volatiles and what their exact functions are. It has been proposed that volatiles represent waste material or a detoxification system of the producing microorganisms (Claeson, 2007). However, from recent studies summarised in part 2 and 3 of this review, it is clear that microbial volatiles can have two major roles in a long-distance interactions in microbial communities as: (i) infochemical molecules affecting the behaviour, population dynamic and gene expression in the responding microorganism, and (ii) competitive tools directly exerting antimicrobial activity, providing an advantage by suppressing or eliminating potential enemies.

Currently, most studies on microbial volatiles are performed in vitro under nutrient rich conditions (Kai et al., 2009; Weise et al., 2012) and may not represent the conditions that prevail in the microbial environment. Furthermore, as indicated by Garbeva et al. (2014a,b), the composition of volatiles produced by a mixture of bacterial species can differ from those produced by each bacterial monoculture.

Soil is a complex, highly diverse and heterogeneous environment; an important characteristic of most soils is the occurrence of air-filled pores. Hence, the gaseous phase forms an important part of the natural surroundings of soil microorganisms. It has been estimated that the area of soil particles covered by microorganisms is less than $1 \%$, implying that the distance between microcolonies of microbial neighbours can be considerable (Young et al., 2008). Compared with diffusible compounds, volatile compounds can travel faster and over longer distances through both the liquid and gaseous phase of the soil (Insam and Seewald, 2010; Effmert et al., 2012), which facilitate the interactions between soil microorganisms. Therefore, volatiles have an important role in the communication and competitiveness between physically separated soil microorganisms (Kai et al., 2009; Effmert et al., 2012; Garbeva et al., 2014a). It is plausible that in soil, dormant microorganisms can sense changes in their environments via emitted volatiles and change their behaviour accordingly and in turn, influence the behaviour of other soil microorganisms (Garbeva et al., 2011). Although several studies have shown that volatile compounds can be used as signalling molecules in soil microbial communication, so far it is unclear how volatiles are perceived as signals by the microorganisms.

To date, little is still known about the regulatory pathways and genes involved in volatile biosynthesis, as well as the possible role of QS in the production of volatiles. Because the production of volatiles is often reported to vary depending on cell density (Weise et al., 2012; Groenhagen et al., 2013), it is tempting to reason that volatiles are regulated by QS. However, there are only few and contradictory reports regarding QS regulation of volatile production. Whereas for hydrogen cyanide in Pseudomonas and Chromobacterium species it was concluded to be QS regulated (Pessi and Haas, 2000; Blom et al., 2011), for $B$. ambifaria, production of volatiles appeared not to be controlled by QS as the volatile profiles of the wild-type and the QS mutant were very similar (Groenhagen et al., 2013). Future challenges are therefore to further elucidate the large chemical diversity of microbial volatiles, to discover regulatory pathways and genes involved in the biosynthesis of volatiles in soil bacteria and fungi, to determine biologically relevant concentrations and to resolve the importance of volatiles in ecosystem processes. Monitoring volatiles may be used as a potential indicator of microbial activity, measuring shifts in community composition in the environment and ultimately for determining the soil health status of agricultural soils.

\section{Conflict of Interest}

The authors declare no conflict of interest.

\section{Acknowledgements}

This work is supported by The Netherlands Organization for Scientific Research (NWO) VIDI personal grant to PG (864.11.015). This is publication 5808 of the Netherlands Institute of Ecology (NIOO-KNAW). We wish to thank the anonymous reviewers for their helpful comments.

\section{References}

Ahmad A, Viljoen AM, Chenia HY. (2014). The impact of plant volatiles on bacterial quorum sensing. Lett Appl Microbiol 60: 8-19. 
Atmosukarto I, Castillo U, Hess WH, Sears J, Strobel G. (2005). Isolation and characterization of Muscodor albus I-41.3s, a volatile antibiotic producing fungus. Plant Sci 169: 854-861.

Bernier SP, Letoffe S, Delepierre M, Ghigo J-M. (2011). Biogenic ammonia modifies antibiotic resistance at a distance in physically separated bacteria. Mol Microbiol 81: 705-716.

Bitas V, Kim H-S, Bennett JW, Kang S. (2013). Sniffing on Microbes: diverse roles of microbial volatile organic compounds in plant health. Mol Plant Microbe Interact 26: 835-843.

Blom D, Fabbri C, Eberl L, Weisskopf L. (2011). Volatile-mediated killing of Arabidopsis thaliana by bacteria is mainly due to hydrogen cyanide. Appl Environ Microbiol 77: 1000-1008.

Brana AF, Rodriguez M, Pahari P, Rohr J, Garcia LA, Blanco G. (2014). Activation and silencing of secondary metabolites in Streptomyces albus and Streptomyces lividans after transformation with cosmids containing the thienamycin gene cluster from Streptomyces cattleya. Arch Microbiol 196: 345-355.

Cepl J, Blahuskova A, Cvrckova F, Markos A. (2014). Ammonia produced by bacterial colonies promotes growth of ampicillin-sensitive Serratia sp by means of antibiotic inactivation. FEMS Microbiol Lett 354: 126-132.

Chernin L, Toklikishvili N, Ovadis M, Kim S, Ben-Ari J, Khmel I et al. (2011). Quorum-sensing quenching by rhizobacterial volatiles. Environ Microbiol Rep 3: 698-704.

Claeson AS, Sandstrom M, Sunesson AL. (2007). Volatile organic compounds (VOCs) emitted from materials collected from buildings affected by microorganisms. J Environ Monit 9: 240-245.

Dandurishvili N, Toklikishvili N, Ovadis M, Eliashvili P, Giorgobiani N, Keshelava R et al. (2011). Broad-range antagonistic rhizobacteria Pseudomonas fluorescens and Serratia plymuthica suppress Agrobacterium crown gall tumours on tomato plants. J Appl Microbiol 110: $341-352$.

Effmert U, Kalderas J, Warnke R, Piechulla B. (2012). Volatile mediated interactions between bacteria and fungi in the soil. J Chem Ecol 38: 665-703.

Farag MA, Zhang H, Ryu C-M. (2013). Dynamic chemical communication between plants and bacteria through airborne signals: induced resistance by bacterial volatiles. J Chem Ecol 39: 1007-1018.

Gallucci MN, Oliva M, Casero C, Dambolena J, Luna A, Zygadlo J et al. (2009). Antimicrobial combined action of terpenes against the food-borne microorganisms Escherichia coli, Staphylococcus aureus and Bacillus cereus. Flavour Fragr J 24: 348-354.

Garbeva P, Hol WHG, Termorshuizen AJ, Kowalchuk GA, de Boer W. (2011). Fungistasis and general soil biostasis - a new synthesis. Soil Biol Biochem 43: 469-477.

Garbeva P, Hordijk C, Gerards S, De Boer W. (2014a). Volatile-mediated interactions between phylogenetically different soil bacteria. Front Microbiol 5: 285-290.

Garbeva P, Hordijk C, Gerards S, de Boer W. (2014b). Volatiles produced by the mycophagous soil bacterium Collimonas. FEMS Microbiol Ecol 87: 639-649.

Groenhagen U, Baumgartner R, Bailly A, Gardiner A, Eberl L, Schulz S et al. (2013). Production of bioactive volatiles by different Burkholderia ambifaria strains. J Chem Ecol 39: 892-906.
Gurtler H, Pedersen R, Anthoni U, Christophersen C, Nielsen PH, Wellington EMH et al. (1994). Albaflavenone, a sesquiterpene ketone with a zizaene skeleton produced by a streptomycete with a new rope morphology. J Antibiot 47: 434-439.

Hagai E, Dvora R, Havkin-Blank T, Zelinger E, Porat Z, Schulz S et al. (2014). Surface-motility induction, attraction and hitchhiking between bacterial species promote dispersal on solid surfaces. ISME $J$ 8: 1147-1151.

Hemaiswarya S, Doble M. (2010). Synergistic interaction of phenylpropanoids with antibiotics against bacteria. J Med Microbiol 59: 1469-1476.

Herrington PR, Craig JT, Chea CY, Sheridan JE. (1985). Inhibition of spore germination by volatiles from Streptomyces griseoruber. Soil Biol Biochem 17: 897-898.

Herrington PR, Craig JT, Sheridan JE. (1987). Methyl vinyl ketone - a volatile fungistatic inhibitor from Streptomyces griseoruber. Soil Biol Biochem 19: 509-512.

Hornby JM, Jensen EC, Lisec AD, Tasto JJ, Jahnke B, Shoemaker R et al. (2001). Quorum sensing in the dimorphic fungus Candida albicans is mediated by farnesol. Appl Environ Microbiol 67: 2982-2992.

Hunziker L, Boenisch D, Groenhagen U, Bailly A, Schulz S, Weisskopf L. (2015). Pseudomonas strains naturally associated with potato plants produce volatiles with high potential for inhibition of Phytophthora infestans. Appl Environ Microbiol 81: 821-830.

Insam H, Seewald MSA. (2010). Volatile organic compounds (VOCs) in soils. Biol Fertil soils 46: 199-213.

Junker RR, Tholl D. (2013). Volatile organic compound mediated interactions at the plant-microbe interface. J Chem Ecol 39: 810-825.

Kai M, Effmert U, Berg G, Piechulla B. (2007). Volatiles of bacterial antagonists inhibit mycelial growth of the plant pathogen Rhizoctonia solani. Arch Microbiol 187: 351-360.

Kai M, Haustein M, Molina F, Petri A, Scholz B, Piechulla B. (2009). Bacterial volatiles and their action potential. Appl Microbiol Biotechnol 81: 1001-1012.

Kim K-S Lee S, Ryu C-M. Interspecific bacterial sensing through airborne signals modulates locomotion and drug resistance. Nat Commun 2013; 4: 1809.

Korpi A, Jarnberg J, Pasanen AL. (2009). Microbial volatile organic compounds. Crit Rev Toxicol 39: 139-193.

Lee HH, Molla MN, Cantor CR, Collins JJ. (2010). Bacterial charity work leads to population-wide resistance. Nature 467: 82-U113.

Lemfack MC, Nickel J, Dunkel M, Preissner R, Piechulla B. (2014). mVOC: a database of microbial volatiles. Nucleic Acids Res 42: D744-D748.

Létoffé S, Audrain B, Bernier SP, Delepierre M, Ghigo J-M. (2014). Aerial exposure to the bacterial volatile compound trimethylamine modifies antibiotic resistance of physically separated bacteria by raising culture medium pH. MBio 5: e00944-13.

Lupetti A, Danesi R, Campa M, Del Tacca M, Kelly S. (2002). Molecular basis of resistance to azole antifungals. Trends Mol Med 8: 76-81.

Lushchak VI. (2001). Oxidative stress and mechanisms of protection against it in bacteria. Biochemistry (Mosc) 66: $476-489$.

Marquez-Villavicencio MdP, Weber B, Witherell RA, Willis DK, Charkowski AO. (2011). The 3-hydroxy-2- 
butanone pathway is required for pectobacterium carotovorum pathogenesis. PLoS One 6: e22974.

Martins M, Henriques M, Azeredo J, Rocha SM, Coimbra MA, Oliveira R. (2007). Morphogenesis control in Candida albicans and Candida dubliniensis through signaling molecules produced by planktonic and biofilm cells. Eukaryot Cell 6: 2429-2436.

Minerdi D, Moretti M, Gilardi G, Barberio C, Gullino ML, Garibaldi A. (2008). Bacterial ectosymbionts and virulence silencing in a Fusarium oxysporum strain. Environ Microbiol 10: 1725-1741.

Minerdi D, Bossi S, Gullino ML, Garibaldi A. (2009). Volatile organic compounds: a potential direct long-distance mechanism for antagonistic action of Fusarium oxysporum strain MSA 35. Environ Microbiol 11: 844-854.

Mitchell AM, Strobel GA, Moore E, Robison R, Sears J. (2010). Volatile antimicrobials from Muscodor crispans, a novel endophytic fungus. Microbiology 156: 270-277.

Molina-Santiago C, Daddaoua A, Fillet S, Duque E, Ramos J-L. (2014). Interspecies signalling: Pseudomonas putida $\backslash$ efflux pump TtgGHI is activated by indole to increase antibiotic resistance. Environm Microbiol 16: 1267-1281.

Moody SC, Zhao B, Lei L, Nelson DR, Mullins JG, Waterman MR et al. (2012). Investigating conservation of the albaflavenone biosynthetic pathway and CYP170 bifunctionality in streptomycetes. FEBS $J$ 279: 1640-1649.

Nemcovic M, Jakubikova L, Viden I, Farkas V. (2008). Induction of conidiation by endogenous volatile compounds in Trichoderma spp. FEMS Microbiol Lett 284: 231-236.

Peñuelas J, Asensio D, Tholl D, Wenke K, Rosenkranz M, Piechulla B et al. (2014). Biogenic volatile emissions from the soil. Plant Cell Environ 37: 1866-1891.

Pessi G, Haas D. (2000). Transcriptional control of the hydrogen cyanide biosynthetic genes henABC by the anaerobic regulator ANR and the quorum-sensing regulators LasR and RhlR in Pseudomonas aeruginosa. $J$ Bacteriol 182: 6940-6949.

Piechulla B, Degenhardt J. (2014). The emerging importance of microbial volatile organic compounds. Plant Cell Environ 37: 811-812.

Rajini KS, Aparna P, Sasikala C, Ramana CV. (2011). Microbial metabolism of pyrazines. Crit Rev Microbiol 37: 99-112.

Romoli R, Papaleo MC, De Pascale D, Tutino ML, Michaud L, LoGiudice et al. (2014). GC-MS volatolomic approach to study the antimicrobial activity of the antarctic bacterium Pseudoalteromonas sp TB41. Metabolomics 10: 42-51.
Schulz S, Dickschat JS. (2007). Bacterial volatiles: the smell of small organisms. Nat Prod Rep 24: 814-842.

Schulz S, Dickschat JS, Kunze B, Wagner-Dobler I, Diestel R, Sasse F. (2010). Biological activity of volatiles from marine and terrestrial bacteria. Mar Drugs 8: 2976-2987.

Seyedmousavi S, Mouton JW, Melchers WJG, Bruggemann RJM, Verweij PE. (2014). The role of azoles in the management of azole-resistant aspergillosis: from the bench to the bedside. Drug Resist Updat 17: $37-50$.

Shatalin K, Shatalina E, Mironov A, Nudler E. (2011). H2S: a universal defense against antibiotics in bacteria. Science 334: 986-990.

Snelders E, RAGHIt Veld, AJMM Rijs, Kema GHJ, Melchers WJG, Verweij PE. (2009). Possible environmental origin of resistance of Aspergillus fumigatus to medical triazoles. Appl Environ Microbiol $\mathbf{7 5}$ 4053-4057.

Strobel GA, Dirkse E, Sears J, Markworth C. (2001). Volatile antimicrobials from Muscodor albus, a novel endophytic fungus. Microbiology 147: 2943-2950.

Takamatsu S, Lin X, Nara A, Komatsu M, Cane DE, Ikeda H. (2011). Characterization of a silent sesquiterpenoid biosynthetic pathway in Streptomyces avermitilis controlling epi-isozizaene albaflavenone biosynthesis and isolation of a new oxidized epiisozizaene metabolite. Microb Biotechnol 4: 184-191.

Trombetta D, Castelli F, Sarpietro MG, Venuti V, Cristani M, Daniele C et al. (2005). Mechanisms of antibacterial action of three monoterpenes. Antimicrob Agents Chemother 49: 2474-2478.

Venkataraman A, Rosenbaum MA, Werner JJ, Winans SC, Angenent LT. (2014). Metabolite transfer with the fermentation product 2,3-butanediol enhances virulence by Pseudomonas aeruginosa. ISME $J$ 8: 1210-1220.

Vespermann A, Kai M, Piechulla B. (2007). Rhizobacterial volatiles affect the growth of fungi and Arabidopsis thaliana. Appl Environ Microbiol 73: 5639-5641.

Weise T, Kai M, Gummesson A, Troeger A, von Reuss S, Piepenborn S et al. (2012). Volatile organic compounds produced by the phytopathogenic bacterium Xanthomonas campestris pv. vesicatoria 85-10. Beilstein J Org Chem 8: 579-596.

Wheatley RE. (2002). The consequences of volatile organic compound mediated bacterial and fungal interactions. Antonie Van Leeuwenhoek 81: 357-364.

Young IM, Crawford JW, Nunan N, Otten W, Spiers A. (2008). Microbial distribution in soils: physics and scaling. In: Sparks DL (ed), Advances in Agronomy vol 100. pp 81-121. 